\section{Great expectancies of emotion}

\author{
Eric A. Salzen
}

Learning and Emotion: A Biological Synthesis. Volume 1, Evolutionary Processes. By Peter J. Livesey. Lawrence Erlbaum:1986. Pp.312. \$32.50, £22.

EMOTION has been variously seen as providing emergency support responses, energizing and response amplification, motivation through incentive and reinforcement, and adaptive instinctive responses, or as being non-functional response disorganization. In this book, Livesey sets out to establish emotion as a system for registering what is or is not relevant for learning. To do this he reviews the evolution of the brain, learning and emotion, with emphasis on species putatively related to the evolutionary line leading to man.

Part I gives an elementary introduction to evolution and a thumbnail sketch of the evolution of man drawn from literature of the 1950 s and 1960 s. Part II, the longest, describes the evolution of the nervous system from amoeba to man, based on literature of the 1960 s and 1970 s. It gives a useful summary for the mammalian brain. Part III critically reviews comparative studies of learning in vertebrates, and covers much the same ground as E.M. Macphail's Brain and Intelligence in Vertebrates (Clarendon, 1982) and Animal Intelligence, edited by L. Weiskrantz (Clarendon, 1985). Livesey, however, is more selective and considers the learning-task performance studies of traditional comparative psychology, Bitterman's work on the systematic variation of a single learning-task, and the theoretical views of Thorpe, Lorenz and Razran on the evolution of learning. Unlike Macphail, Livesey concludes that there has been a qualitative change in learning processes and that "expectancy" is a crucial concept in this respect.

In the fourth and final part, Livesey gives his views on the contribution of affect to this evolution of learning. He begins by defining emotion as "a reactive state evolving from the confluence of the affects of reward and punishment and of expectancies generated through associative learning" (p. 231). After a brief and selective consideration of some literature on emotion, Livesey presents his scheme of the evolution of affect. He claims that: (1) the brain reward/punishment system postulated by Olds does indeed promote feelings or signals of the effectiveness of behaviour and is present in all vertebrates; (2) the evolution of learning involving the anticipation of outcomes and the matching of expectancies and outcomes pro-

vides a new feeling system that coincides with the evolution of mammals; and (3) these feelings can in turn modify expectancies and be externalized as social signals of emotional states.

Livesey thus effectively matches his earlier definition of emotion, no doubt to his satisfaction and pleasure. His thesis implies that the evolution of emotion depended on the evolution of learned expectancies. He also proposes that the function of emotion is to feed back and influence expectancies and so act as a "teaching mechanism". If emotion guides learning as Livesey implies, one wonders what guided Oscar Wilde when, in The Picture of Dorian Gray, he wrote: "The advantage of the emotions is that they lead us astray". Livesey's theory fails to account for the evolution of emotional behaviours which are present throughout the vertebrates and are especially evident in birds,

\section{Universal classics}

\section{Michael Rowan-Robinson}

Cosmological Constants: Papers in Modern Cosmology. Edited by Jeremy Bernstein and Gerald Feinberg. Columbia University Press:1986. Pp.328. $\$ 38$.

THE twentieth century has seen the most remarkable development in our understanding of the Universe, and Bernstein and Feinberg's idea of collecting together some of the classic papers of the great modern age of cosmology was an excellent one. Most of the really important ones are here. The first tentative speculations by Einstein in 1917 about a homogeneous and isotropic, but static, universe; de Sitter's disturbing revelation (also of 1917) that Einstein's equations allowed the possibility of an expanding universe; Friedmann's spectacular solution of the cosmological problem in general relativity (1922 and 1924), giving us the models which have formed the basis for cosmological studies ever since; Robertson's (1929) elegant reformulation of these solutions with far more satisfactory underlying assumptions. And then there are the observations, as usual limping along behind theory, with Hubble's 1929 statement, on the basis of the flimsiest of evidence, of his immortal law.

There is quite a gap until the hot Big Bang of Gamov (1948) and Alpher and Herman (1949), and then another long gap until those two remarkable papers which appeared back-to-back in Astrophysical Journal in 1965: the two-page announcement by Penzias and Wilson of the discovery of the microwave background, with virtually no realization of the significance of the discovery, and the hasty interpretation by Dicke, Peebles, Roll whose evolutionary origin and brain structure are quite different from those of mammals, to whom, apparently, emotion sensu strictu is restricted.

Students might find Parts I-III of this book valuable in appreciating the theoretical propositions of Part IV. The specialist reader, however, might have preferred the relevant facts to have been referenced and integrated into the presentation of the theory. In Vol. 2 of the work (which, I'm told, will not be published until 1988 or so), Livesey proposes to identify the neural systems for directing attention to relevant stimuli and for organizing appropriate behaviour on subsequent encounters, with special reference to the role of the limbic system in the mediation of this learning.

Eric A. Salzen is Anderson Professor in the Department of Psychology, University of Aberdeen, Old Aberdeen AB92UB, UK.

and Wilkinson, pipped to the discovery by perhaps a year.

Finally there is a batch of papers on the new synthesis of particle physics and cosmology, though it is probably too soon to say that any of the work is on the right lines. We have the insight of Sakharov (1967), in his happier days, on baryon non-conservation, and its realization in grand unified theories by Yoshimura, Weinberg and others; Kibble (1976) on cosmic strings; Preskill (1979) on the monopole problem; and Guth's proposal (1981) of the inflationary universe.

Not all of the papers included are of the same standing as those mentioned above. The editors have chosen quite the wrong one by Sandage on the Hubble constant a minor 1968 paper, using globular clusters, instead of that of 1956 in which he revised Baade's distance scale by a factor of nearly four to arrive at a Hubble constant of 75. The famous 1941 paper by McKellar, measuring a background temperature of $2.3 \mathrm{~K}$, is absent, as are the near-miss papers of Hoyle and Tayler (1964) and Doroshkevich and Novikov (1964) on helium production, and there is nothing from the ever-influential Zeldovich.

The book has been produced in cameraready format, so I do not understand why the editors opted to retype every paper rather than reproduce them in facsimile. I think most readers would have been willing to do a bit of work on the notation to get that breath of history. But even if it reads like the ultimate summer school proceedings, this anthology will be irresistible to all with a serious interest in cosmology and the history of science.

Michael Rowan-Robinson is Reader in Astronomy in the School of Mathematical Sciences, Oueen Mary College, Mile End Road, London E1 4NS, UK, and author of The Cosmological Distance Ladder (W.H. Freeman, 1985). 